\title{
Use of alternative bioassays to explore the impact of pyrethroid resistance on LLIN efficacy
}

\author{
Marissa K. Grossman ${ }^{1 *}$, Shüné V. Oliver ${ }^{2,3}$, Basil D. Brooke ${ }^{2}$ and Matthew B. Thomas
}

\begin{abstract}
Background: There is substantial concern that the spread of insecticide resistance will render long-lasting insecticide-treated nets (LLINs) ineffective. However, there is limited evidence supporting a clear association between insecticide resistance and malaria incidence or prevalence in the field. We suggest that one reason for this disconnect is that the standard WHO assays used in surveillance to classify mosquito populations as resistant are not designed to determine how resistance might impact LLIN efficacy. The standard assays expose young, unfed female mosquitoes to a diagnostic insecticide dose in a single, forced exposure, whereas in the field, mosquitoes vary in their age, blood-feeding status, and the frequency or intensity of LLIN exposure. These more realistic conditions could ultimately impact the capacity of "resistant" mosquitoes to transmit malaria.
\end{abstract}

Methods: Here, we test this hypothesis using two different assays that allow female mosquitoes to contact a LLIN as they host-seek and blood-feed. We quantified mortality after both single and multiple exposures, using seven different strains of Anopheles ranging in pyrethroid resistance intensity.

Results: We found that strains classified as $1 \times$-resistant to the pyrethroid insecticide deltamethrin in the standard WHO assay exhibited $>90 \%$ mortality over $24 \mathrm{~h}$ following more realistic LLIN contact. Mosquitoes that were able to blood-feed had increased survival compared to their unfed counterparts, but none of the $1 \times$-resistant strains survived for 12 days post-exposure (the typical period for malaria parasite development within the mosquito). Mosquitoes that were $5 x$ - and $10 x$-resistant (i.e. moderate or high intensity resistance based on the WHO assays) survived a single LLIN exposure well. However, only about 2-3\% of these mosquitoes survived multiple exposures over the course of 12 days and successfully blood-fed during the last exposure.

Conclusions: These results suggest that the standard assays provide limited insight into how resistance might impact LLIN efficacy. In our laboratory setting, there appears little functional consequence of $1 \times$-resistance and even mosquitoes with moderate $(5 x)$ or high $(10 x)$ intensity resistance can suffer substantial reduction in transmission potential. Monitoring efforts should focus on better characterizing intensity of resistance to inform resistance management strategies and prioritize deployment of next generation vector control products.

Keywords: Insecticide resistance, Anopheles, Malaria, Pyrethroids

*Correspondence: mkgrossman@gmail.com

${ }^{1}$ Department of Entomology, Pennsylvania State University, University Park, PA, USA

Full list of author information is available at the end of the article

c) The Author(s) 2020. This article is licensed under a Creative Commons Attribution 4.0 International License, which permits use, sharing, adaptation, distribution and reproduction in any medium or format, as long as you give appropriate credit to the original author(s) and the source, provide a link to the Creative Commons licence, and indicate if changes were made. The images or other third party material in this article are included in the article's Creative Commons licence, unless indicated otherwise in a credit line to the material. If material is not included in the article's Creative Commons licence and your intended use is not permitted by statutory regulation or exceeds the permitted use, you will need to obtain permission directly from the copyright holder. To view a copy of this licence, visit http://creativecommons.org/licenses/by/4.0/. The Creative Commons Public Domain Dedication waiver (http://creativecommons.org/publicdomain/zero/1.0/) applies to the data made available in this article, unless otherwise stated in a credit line to the data. 


\section{Background}

The prevalence of insecticide resistance in malaria vector populations has been increasing steadily over the past 15 years [1], leading to concerns over widespread failure of insecticide-based vector control measures such as long-lasting insecticidal nets (LLIN) [2, 3]. However, despite these concerns, evidence of LLIN control failure due to resistance is mixed. The most compelling data derive from a randomized controlled trial in an area of high pyrethroid resistance in Tanzania, which showed that LLINs containing a synergist that inhibits the detoxification of pyrethroids by resistant mosquitoes provided improved control of malaria transmission compared with a standard LLIN [4]. Other studies, however, have found that LLINs are still more effective in preventing both clinical and subclinical malaria infections than untreated nets in areas with high pyrethroid resistance [5-8], including a 4-year WHO-coordinated 5-country cohort study that found no association between pyrethroid resistance and malaria incidence or prevalence in the field [9]. While observational studies need to be treated with caution as they cannot control for various factors and tend to only assess personal protection (a modeling analysis suggests that there could be loss of community protection even if levels of personal protection from LLINs remains high [10]), there are clearly complexities in interpreting the epidemiological consequences of resistance [11, 12].

There are a number of reasons why insecticide resistance might not reduce the apparent effectiveness of LLINs. First, LLINs provide a physical barrier that can reduce biting rate regardless of insecticidal activity. Secondly, there may be sub-lethal effects to insecticide exposure, so that when a resistant mosquito contacts insecticide, it might experience a decrease in its ability to blood-feed and host-seek [11], or even incubate the parasite $[13,14]$. Thirdly, alleles that confer resistance in mosquitoes might also reduce vector competence in the absence of insecticide exposure [15]. Fourthly, there might be fitness costs to resistance [16-18], which would impact the resistant population's survival (and therefore they may not survive long enough to potentially transmit the parasite), though this is not always the case [19]. This diversity of effects illustrates the potential for complex interactions between resistance and overall vectorial capacity of mosquito populations [20].

One additional explanation for why resistance has not substantially impacted control is that we might not have reached a tipping point at which resistance is intense enough to hinder control efforts [12]. This possibility raises questions over the way in which resistance is characterized in the field. The standard WHO resistance assay consists of placing up to 25 female mosquitoes in a plastic tube lined with insecticide-treated paper for one hour and then evaluating mortality 24 hours after exposure [21]. The assay uses a diagnostic concentration of insecticide that is twice the lowest concentration determined to cause $100 \%$ mortality of a susceptible strain [21]. If the test mosquitoes display less than $90 \%$ mortality to this concentration, the population is characterized as resistant. The assay is designed as a surveillance tool to detect the emergence of resistance, and there are numerous efforts underway that collate prevalence data to illustrate the spatial and temporal distribution of resistance [22-24]. However, demonstrating the presence of resistance in field populations is not the same as demonstrating functional significance. In particular, the WHO tube assay exposes young (3-5 day-old), non-blood-fed, and non-infectious mosquitoes to a relatively low diagnostic dose of insecticide. In the field, the mosquitoes responsible for transmission are at least two weeks-old, have had at least one blood meal, and might well have experienced multiple exposures to higher concentrations of insecticides through repeated contact with LLINs. These differences could matter since it has been shown that phenotypic expression of resistance declines with mosquito age [25-27], can be affected by blood-feeding status $[28,29]$, and can decrease with multiple exposures [30]. Recently, WHO expanded the scope of the standard tube assay to measure the intensity of resistance by increasing the diagnostic dose to $5 \times$ and $10 \times$ [21]. While these doses add more information on the nature of resistance, the operational relevance of moderate $(5 \times)$ or high $(10 \times)$ intensity resistance in a tube test remains unclear.

While the WHO has developed the cone test and the tunnel test to directly assess the bioefficacy of LLINs [31], these tests also fail to better approximate field conditions. The cone test, which exposes groups of five mosquitoes directly to an LLIN, also dictates the use of young, nonblood-fed mosquitoes, and forces exposure through a very confined area instead of allowing the mosquito to naturally contact the net. The tunnel test, on the other hand, allows the mosquito to host-seek and blood-feed, though it uses a rodent host, which is not the preferred host of the anthropophilic Anopheles malaria vectors [32]. At present, there are no existing tools that can fully evaluate the response of resistant mosquitoes as they naturally contact a LLIN.

Here, we quantify the impacts of a standard LLIN on strains of Anopheles spp. with different resistance intensities under assay conditions that allow for single or multiple contacts with the LLIN as the mosquitoes host-seek and blood-feed. The aim is to better understand the functional significance of $1 \times, 5 \times$ or $10 \times$ resistance in terms of likely efficacy failure of an LLIN. 


\section{Methods}

\section{Strain characterization}

We used 7 laboratory strains of Anopheles spp. ranging from fully susceptible to $10 \times$ pyrethroid-resistant according to the WHO tube assays (Table 1). All strains were maintained at the National Institute for Communicable Diseases in Johannesburg, South Africa, with strains maintained as per Hunt et al. [33]. Further strain details are provided in Venter et al. [34].

\section{Experimental design \\ Experiment 1}

As described above, the standard WHO resistance assay involves forced exposure of mosquitoes to a diagnostic dose of insecticide. To simulate more realistic exposure conditions, we suspended either an untreated net or a Permanet 2.0 (a polyester LLIN coated with deltamethrin at $1.8 \mathrm{~g} / \mathrm{kg}$ ) down the middle of a BugDorm2120 Insect Rearing Tent, dividing the tent into two sections (Fig. 1a). Exposures were performed at $25 \pm 2{ }^{\circ} \mathrm{C}$ and at a relative humidity of $80 \pm 5 \%$. A single human host placed her arm inside the tent on one side of the Permanet, pressing her arm against the side of the net, simulating what might happen if a person slept touching an LLIN. We released 20-30 mosquitoes into the tent on the other side of the net from the human host. The net made a functional barrier between the mosquitoes and the host; the only way the mosquito could bite the host was through the net. Mosquitoes that were released into the tent were allowed to host-seek and blood-feed for $20 \mathrm{~min}$. Previous research found that when mosquitoes encounter a LLIN, the majority of activity occurs in the first $10 \mathrm{~min}$, with minimal activity after $30 \mathrm{~min}$ [35]. Pilot tests with our experimental set-up revealed that activity greatly reduced after $20 \mathrm{~min}$, so we chose that as the experimental time. During the assay, we counted the number of individual mosquitoes that engaged in host-seeking behavior, defined as flying toward the host and contacting the net,

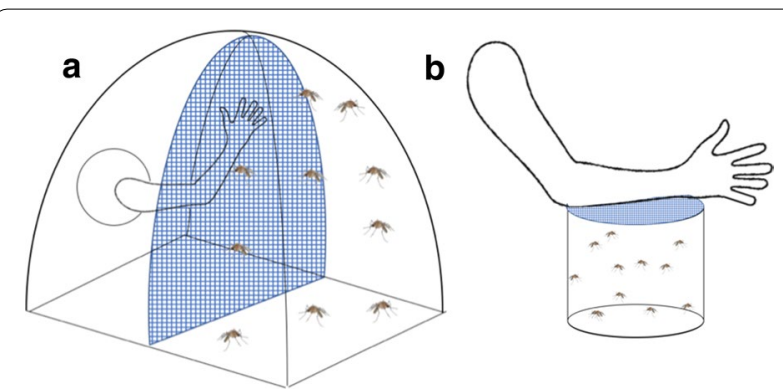

Fig. 1 Experimental setup. a Experiment 1, the "tent" assay: a Permanet 2.0 divided a BugDorm2120 Insect Rearing Tent into two sections. A human host placed her arm inside of the tent and pressed it against the net, allowing mosquitoes on the other side of the net to obtain a bloodmeal through the net. b Experiment 2, the "cup" assay: a $160 z$ paper cup was covered with a Permanet 2.0. Mosquitoes were released into the cup through a small hole in the net, and the human host placed her arm on top of the net, allowing mosquitoes to obtain a blood meal through the net

regardless of time spent on the net. It was possible to track individual mosquitoes due to limited flight activity. At the end of $20 \mathrm{~min}$, all mosquitoes were separated by blood-fed status and transferred into holding cups with $10 \%$ sucrose solution. Initial mortality, 24-h mortality, and subsequent daily mortality was recorded for 12 days for both blood-fed and non-blood-fed mosquitoes. Surviving mosquitoes at day 12 post-assay were re-released into the tent and allowed to host-seek and blood-feed using the same methods. The timing of this second exposure was designed to coincide with the typical time taken for malaria parasites to complete development within the mosquito (the extrinsic incubation period, EIP) if the mosquito had acquired parasites during the first blood meal [36]. Only those mosquitoes that survive across the EIP and take at least two blood meals can transmit malaria. All assays were conducted in the dark using a red light. The control assays with the untreated net were always conducted first to minimize any risk of insecticide

Table 1 Strain characterization

\begin{tabular}{|c|c|c|c|c|c|}
\hline Species & Strain & Origin & Date colonized & $\begin{array}{l}\text { Resistance intensity to } \\
\text { deltamethrin }\end{array}$ & Mortality (\%) \\
\hline An. arabiensis & KGB & Kanyemba, Zimbabwe & 1975 & Susceptible reference & - \\
\hline An. funestus & FANG & Calueque, Angola & 2002 & Susceptible reference & - \\
\hline An. arabiensis & SENN & Sennar, Sudan & 1980 & Susceptible & $>98$ \\
\hline An. arabiensis & SENN-DDT & Sennar, Sudan & Selected since 1995 & $1 \times$ & 54 \\
\hline An. gambiae & TONGS & Tongon, Côte D’Ivoire & 2010 & $1 \times$ & 91 \\
\hline An. funestus & FUMOZ & Maputo, Mozambique & 2000 & $5 \times$ & 8 \\
\hline An. funestus & FUMOZ-R & Maputo, Mozambique & 2001 & $10 \times$ & 4 \\
\hline
\end{tabular}

Resistance intensity to the pyrethroid deltamethrin was previously measured under standard laboratory conditions with the WHO tube assays [34]

a $24 \mathrm{~h}$ post-exposure to $1 \times$ diagnostic dose of deltamethrin 
contamination of the human host, who was the same for all experiments and replicates. Four replicates were conducted for each strain and each condition (untreated net vs LLIN). Experiments occurred during October 2017, while tests in Experiment 2 were conducted in February 2018.

\section{Experiment 2}

The first experiment revealed low recruitment to the host in some mosquito strains. Low recruitment, and therefore low contact with the LLIN, complicated comparison of mortality across strains (note, however, that spatial repellency and/or contact irritancy can be functional properties of an LLIN [37]). In an attempt to address this problem, we conducted a follow-up experiment using much smaller paper cups, measuring $475 \mathrm{ml}$, as the enclosure (Fig. 1b). Netting was used to cover the opening of the cup and the arm of a volunteer was positioned on top of the netting. Our aim was to allow mosquitoes to contact the netting naturally during host-seeking and blood-feeding as before, but to strengthen the host cues so that the proportion of responders was increased. The same basic procedures were followed as in experiment one, with a few modifications: (i) total experimental time was only 15 min instead of 20 because pilot tests revealed the mosquitoes recruited much faster in the smaller space; (ii) contact with the net, without bloodfeeding, could not be seen due to experimental set-up, so only the number of blood-fed mosquitoes was recorded; (iii) the assay was conducted every 3 days for the 12-day experimental period instead of just day one and day 12 to simulate contact with LLINs as mosquitoes attempt to blood-feed across sequential gonotrophic cycles; and (iv) only SENN-DDT, FUMOZ and FUMOZ-R were used for the experiment since the previous assay showed that the other strains suffered close to $100 \%$ mortality rapidly after a single exposure. The experiment included four replicate cups per strain, with $20-25$ mosquitoes per cup.

\section{Analysis}

A Chi-square test of independence was used to assess the differences in host-seeking and blood-feeding between the LLIN and the untreated net for each mosquito strain. The number of mosquitoes that engaged in host-seeking behavior was defined as the number that contacted the net for any amount of time. To determine the probability of mortality following net exposure, a generalized linear mixed model with a binomial distribution was used with resistance status and treatment (untreated net/LLIN) as predictors. Resistant status was defined as a factor with the categories $0,1,5$ and 10 to indicate resistance intensity with 0 representing the susceptible strains (KGB, FANG and SENN). Strain was used as a random effect to account for multiple replicates.

Kaplan-Meir survival curves, stratified by treatment, were created to visualize differences in survival following net exposure and a log-rank test was used to determine significant differences in survival between the LLIN and the untreated net. Additionally, Cox proportional hazard models were used to assess the mortality rates given treatment, blood-feeding, and resistance status.

\section{Results \\ Experiment 1: Tent assay Host-seeking and blood-feeding}

The proportion of mosquitoes that engaged in hostseeking behavior significantly decreased on the LLIN compared to the untreated net for KGB (Fig. $2 ; X^{2}=27.5$, $d f=1, \quad P \leq 0.0001)$ and FUMOZ $\left(x^{2}=11.5, \quad d f=1\right.$, $P=0.0007)$. However, there was significantly more host-seeking on the LLIN for SENN $\left(\chi^{2}=16.3, d f=1\right.$, $P<0.0001)$, TONGS $\left(x^{2}=28.4, d f=1, P<0.0001\right)$ and

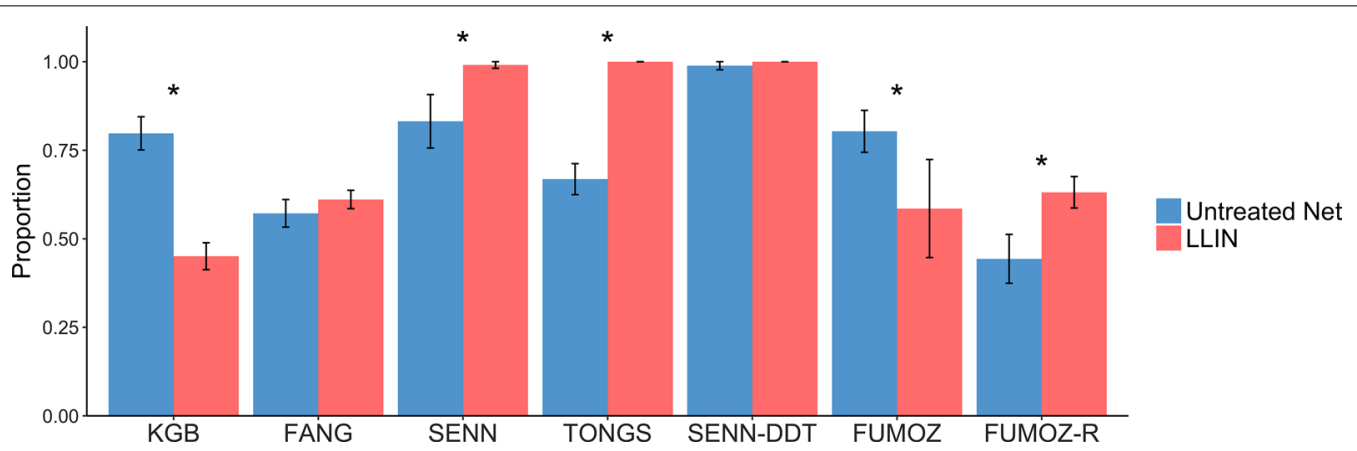

Fig. 2 Host-seeking behavior of mosquitoes in the "tent" assay. The bars show the mean ( \pm standard error, SE) proportion of mosquitoes contacting either an untreated net or an LLIN when attempting to feed on a host arm placed adjacent to the net. Mosquito strains on the $x$-axis are arranged by increasing resistance status. KGB, FANG and SENN are susceptible; TONGS and SENN-DDT are considered $1 \times$ resistant; FUMOZ is $5 \times$; FUMOZ-R is $10 \times$. Statistical significance at the alpha level of 0.05 is marked with an * 
FUMOZ-R $\left(x^{2}=7.1, d f=1, P=0.008\right)$, while there was no difference in host-seeking between the LLIN and untreated net for SENN-DDT $\left(\chi^{2}=0.01, d f=1, P=0.92\right)$. FUMOZ-R and FUMOZ displayed the same amount of host-seeking behavior on the LLIN $\left(\chi^{2}=0.362, d f=1\right.$, $P=0.547$ ), yet the proportion of FUMOZ-R contacting the untreated net was significantly less than that of FUMOZ $\left(\chi^{2}=38.7, d f=1, P<0.0001\right)$. The other paired lines, SENN and SENN-DDT also showed differences in host-seeking behavior on the untreated net only, with a higher proportion of SENN-DDT contacting the untreated net than SENN $\left(\chi^{2}=12.4, d f=1, P=0.0004\right)$.

All mosquito lines, except for FUMOZ-R, bloodfed less when exposed to the LLIN compared to the untreated net, although this decrease was only significant for KGB (Fig. 3; $X^{2}=21.1, d f=1, P<0.0001$ ), SENN-DDT $\left(x^{2}=14.5, d f=1, P=0.0001\right)$ and FUMOZ $\left(\chi^{2}=12.4\right.$, $d f=1, P=0.0004)$. FUMOZ-R, however, blood-fed significantly more through a LLIN than through an untreated net $\left(\chi^{2}=52.2, d f=1, P<0.0001\right)$.

\section{Short-term mortality and extended survival following contact with a net}

For all mosquito lines there was negligible 24-h mortality following exposure to an untreated net (range 0-12\%). The susceptible strains, KGB, SENN and FANG, displayed $97 \pm 1.7 \%$ (KGB) and 100\% (SENN and FANG) mortality at $24 \mathrm{~h}$ post-contact with the LLIN. TONGS and SENNDDT, $1 \times$ resistant strains according to the WHO tube assay, had $93.5 \pm 1.6 \%$ mortality and $84.9 \pm 6.4 \%$ mortality, respectively (Fig. 4). However, the level of mortality was dependent on whether mosquitoes had blood-fed during the LLIN exposure or not. Mortality of bloodfed TONGS and SENN-DDT was $86.7 \pm 3.3 \%$ and $80.3 \pm 7.4 \%$, respectively, compared to $100 \%$ mortality for those that did not blood-feed (Fig. 5). The more resistant FUMOZ and FUMOZ-R exhibited substantially lower overall mortality $(16.4 \pm 5.5 \%$ and $4.8 \pm 1.9 \%$, respectively). Again, mosquitoes that had blood-fed suffered lower mortality than those that had not taken a blood meal $(7.6 \pm 5.2 \%$ compared with $24.9 \pm 9.3 \%$ for FUMOZ and $0 \%$ compared with $8.9 \pm 3.4 \%$ for FUMOZ-R).

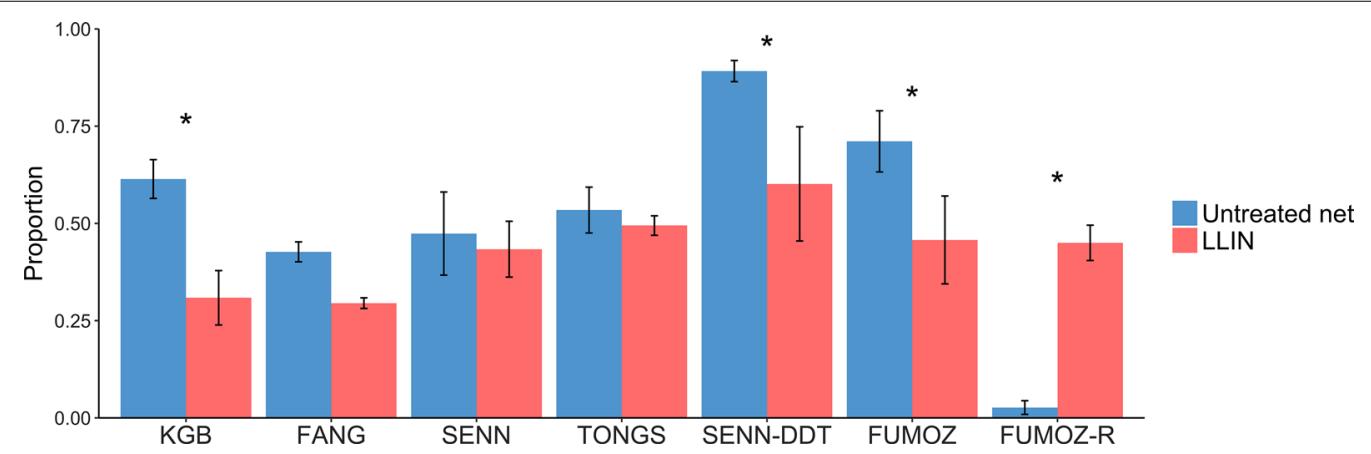

Fig. 3 Blood-feeding behavior of mosquitoes in the "tent" assay. The bars show the mean ( \pm standard error, SE) proportion of mosquitoes that contacted either a LLIN or an untreated net and successfully took a blood meal from a human host arm. Mosquito strains on the $x$-axis are arranged by increasing resistance status. KGB, FANG, and SENN are susceptible; TONGS and SENN-DDT are considered $1 \times$ resistant; FUMOZ is $5 \times$; FUMOZ-R is $10 \times$. Statistical significance at the alpha level of 0.05 is marked with an *

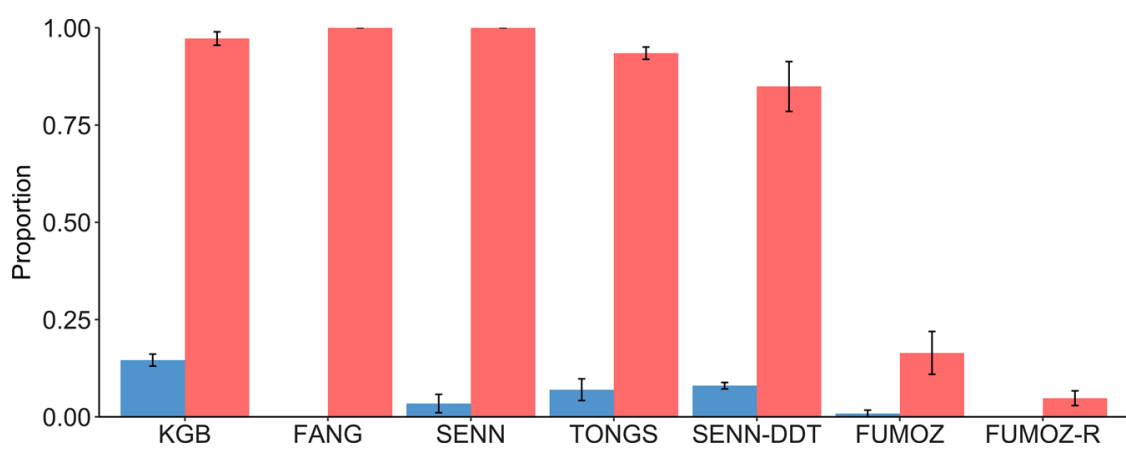

Fig. 4 Mortality 24 hours post-exposure. The bars show the mean ( \pm standard error, SE) proportion of mosquitoes that died following exposure to either an LLIN or an untreated net. Mosquito strains on the $x$-axis are arranged by increasing resistance status. KGB, FANG, and SENN are susceptible; TONGS and SENN-DDT are considered $1 \times$ resistant; FUMOZ is $5 \times$; FUMOZ-R is $10 x$ 


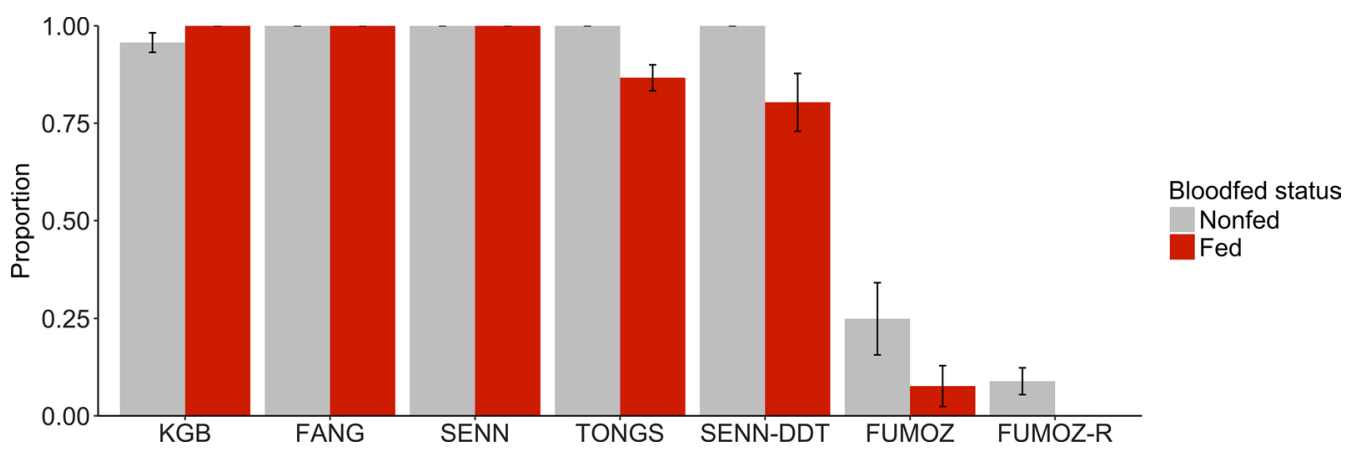

Fig. 5 Mortality 24 hours post-exposure on the LLIN based on blood-fed status. The bars show the mean ( \pm standard error, SE) proportion of mosquitoes that died following either a successful or unsuccessful attempt to blood-feed on a human host arm while being exposed to an LLIN. Mosquito strains on the $x$-axis are arranged by increasing resistance status. KGB, FANG, and SENN are susceptible; TONGS and SENN-DDT are considered $1 \times$ resistant; FUMOZ is $5 \times$; FUMOZ-R is $10 \times$

Table 2 General linear mixed effect model assessing the impact of resistance intensity and the experimental treatment on mosquito mortality

\begin{tabular}{lllll}
\hline Fixed effects & Estimate & SE & Z-value & $P$-value \\
\hline Intercept & -2.39 & 0.24 & -9.84 & $<0.0001$ \\
LLIN & 5.81 & 0.31 & 18.65 & $<0.0001$ \\
1X Resistance & -1.02 & 0.39 & -2.62 & 0.0087 \\
$5 \times$ Resistance & -5.00 & 0.48 & -10.41 & $<0.0001$ \\
10X Resistance & -6.38 & 0.58 & -10.99 & $<0.0001$ \\
\hline
\end{tabular}

Notes: Resistance was defined as a factor, with the levels starting at susceptible (the reference) to $1 \times, 5 \times$ and $10 \times$. Experimental treatment was either the LLIN or the untreated net, which was the reference. Mosquito strain was used a random effect to account for multiple replicates per strain $(n=54$, Groups $=7$, Variance $=0.064$, Std. Dev $=0.253$ )

Abbreviation: $\mathrm{SE}$, standard error

The odds of mosquito mortality after one exposure to the LLIN were 2.8 (95\% CI: $1.2-6.5$ ) lower for $1 \times$ resistant mosquitoes compared to susceptible, 149 (95\% CI: 53.6-459) times lower for $5 \times$ resistant mosquitoes, and 592 times (95\% CI: 189-2194) lower for 10× mosquitoes compared to susceptible, indicating that resistance, as classified by the WHO criteria, does increase survival dramatically (Table 2). Additionally, the odds of mortality of any mosquito following contact with an LLIN, even after accounting for resistance, were 332 (95\% CI: 186-635) times higher than following contact with an untreated net, indicating that the insecticide still has an appreciable effect even in the presence of resistance (Table 2).

Over the course of the entire 12-day experimental period, the overall mortality (regardless of intensity of resistance) for SENN-DDT, FUMOZ and FUMOZ-R was 2.0 (95\% CI: 1.6-2.5) times higher if exposed to the LLIN than the untreated net (Fig. 6; Cox PH, $z=6.27$,
$P<0.0001)$. On the LLIN, SENN-DDT suffered a mortality rate that was 11.7 times (95\% CI: 7.7-17.7) higher than FUMOZ (Cox PH, $z=-11.61, P<0.0001$ ) and 29.1 (95\% CI: 17.8-47.7) times higher than FUMOZ-R (Cox $\mathrm{PH}, z=-13.45, P<0.0001)$.

Of the 6 strains tested, only FUMOZ and FUMOZ-R survived all 12 days post-exposure (to reach 17 days-old) when exposed to the LLIN (Fig. 6). Of the $64.3 \pm 6.1 \%$ of FUMOZ and $85.1 \pm 7.1 \%$ of FUMOZ-R that survived 12-days post LLIN exposure, only $58.8 \pm 9.8 \%$ of FUMOZ and $40.3 \pm 7.6 \%$ of FUMOZ-R contacted the net with only $30.4 \pm 6.5 \%$ of FUMOZ and $7.8 \pm 3.3 \%$ of FUMOZ$\mathrm{R}$ blood-feeding during the second exposure assay. These low rates may be due to age rather than previous exposure: when 17-day-old FUMOZ-R mosquitoes that had no previous exposure were used for the assay on the LLIN, only $20.6 \pm 5.3 \%$ contacted the net and $3.7 \pm 0.07 \%$ blood-fed.

\section{Experiment 2: Cup assay Blood-feeding}

The cup assay increased the proportion of mosquitoes that blood-fed compared to the tent assay on both the LLIN and untreated net for SENN-DDT and FUMOZ$\mathrm{R}$ (Fig. 7). There were no differences between the proportion of mosquitoes that blood-fed on the LLIN compared to the untreated net for the cup assay except for FUMOZ-R, which blood-fed significantly less on the LLIN than on the untreated net $\left(\chi^{2}=10.94, d f=1\right.$, $P=0.0009$ ). Contact rates with the nets could not be observed due to the nature of the cup assay (the arm placed on top of the paper cup obscured behavioral observations). 

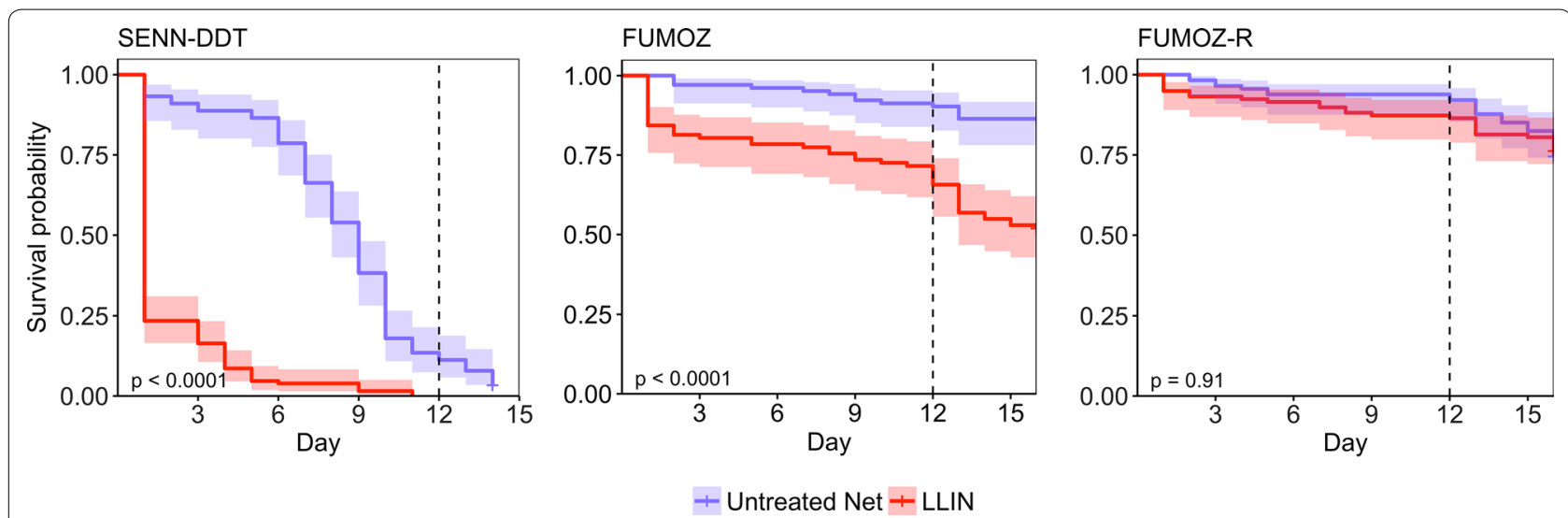

Fig. 6 Survival post-exposure. Exposures occurred on day 0 and day 12, although only FUMOZ and FUMOZ-R were exposed on day 12 due to poor survival of SENN-DDT. Shaded areas represent $95 \%$ confidence intervals. The $P$-value listed is based on the log-rank test to determine differences in the survival curves for the untreated net and LLIN

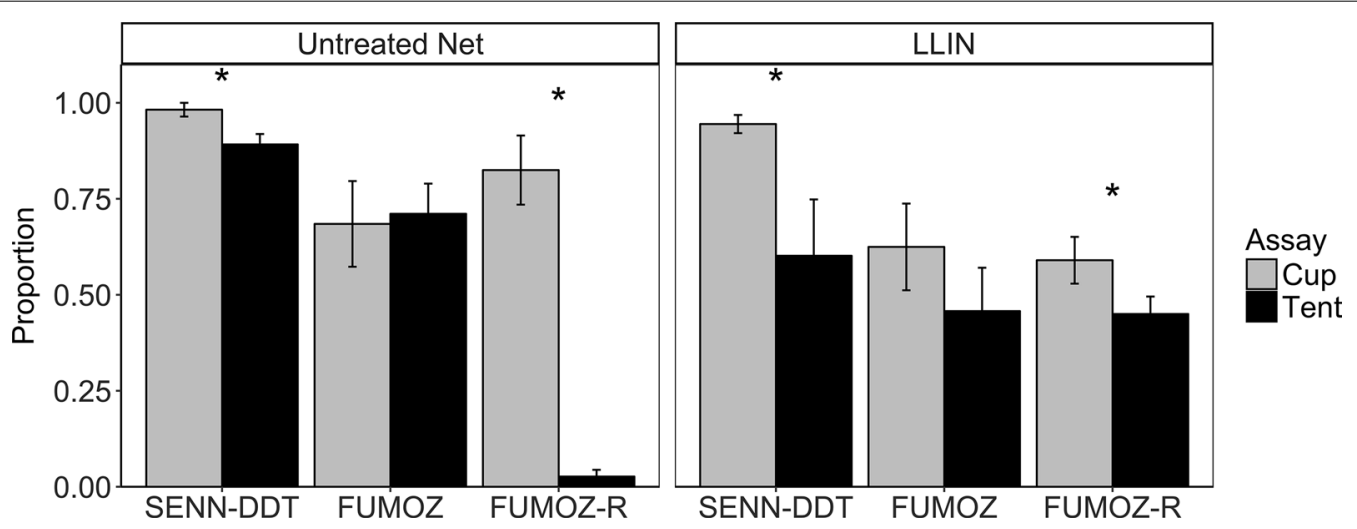

Fig. 7 Blood-feeding behavior of mosquitoes in both the tent assay and the cup assay. The bars show the mean ( \pm standard error, SE) proportion of mosquitoes that contacted either a LLIN or an untreated net and successfully took a blood meal from a human host arm. Mosquito strains on the $x$-axis are arranged by increasing resistance status. KGB, FANG, and SENN are susceptible; TONGS and SENN-DDT are considered $1 \times$ resistant; FUMOZ is $5 \times$; FUMOZ-R is $10 x$. Statistical significance at the alpha level of 0.05 is marked with an *

\section{Short-term mortality and extended survival following contact with a net}

The proportion of mosquitoes that died $24 \mathrm{~h}$ after exposure to the LLIN in the cup assay was $81.1 \%( \pm 7.5 \%)$ for SENN-DDT, 55.7\% $( \pm 12.7 \%)$ for FUMOZ, and $27.1 \%$ $( \pm 11.4 \%)$ for FUMOZ-R. All strains exhibited little to no mortality 24 hours following exposure to an untreated net.

SENN-DDT, FUMOZ, and FUMOZ-R experienced 5 total exposures on either the untreated net or LLIN over the course of 12 days. All strains suffered significantly higher mortality on the LLIN compared to the untreated net (Fig. 8; SENN-DDT: Log-rank test, $\chi^{2}=180$, $P<0.0001$, FUMOZ: Log-rank test, $\chi^{2}=156, P<0.0001$; FUMOZ-R: Log-rank test, $\left.X^{2}=82.1, P<0.0001\right)$. Controlling for the effect of the LLIN and the mosquito strain, mosquitoes that blood-fed at least once during the 5 exposures had a significantly reduced mortality rate compared to those that did not feed at all $(\mathrm{Cox} \mathrm{PH}$, Hazard Ratio $=0.28$, 95\% CI: 0.21-0.36, $P<0.0001$ ). When accounting for blood-feeding, the mortality rate of all strains was 10.5 times higher on the LLIN than the untreated net (Cox PH, Hazard Ratio $=10.52$, 95\% CI: $8.12-13.58, P<0.0001)$. Additionally, the mortality rate of FUMOZ and FUMOZ-R was 3.3 and 5.6 times, respectively, lower than SENN-DDT, accounting for LLIN exposure and blood-feeding (Cox PH; FUMOZ; Hazard Ratio $=0.31,95 \%$ CI: 0.24-0.40, $P<0.0001$; FUMOZ-R: Hazard Ratio $=0.18$, 95\% CI: 0.14-0.23, $P<0.0001$ ).

The proportion of each population that was able to survive the 12-day interval between the first and last exposure on the LLIN and blood-feed during both exposures was minimal, ranging between $0.9-3.2 \%$ (Table 3). This was significantly smaller than the proportion that 


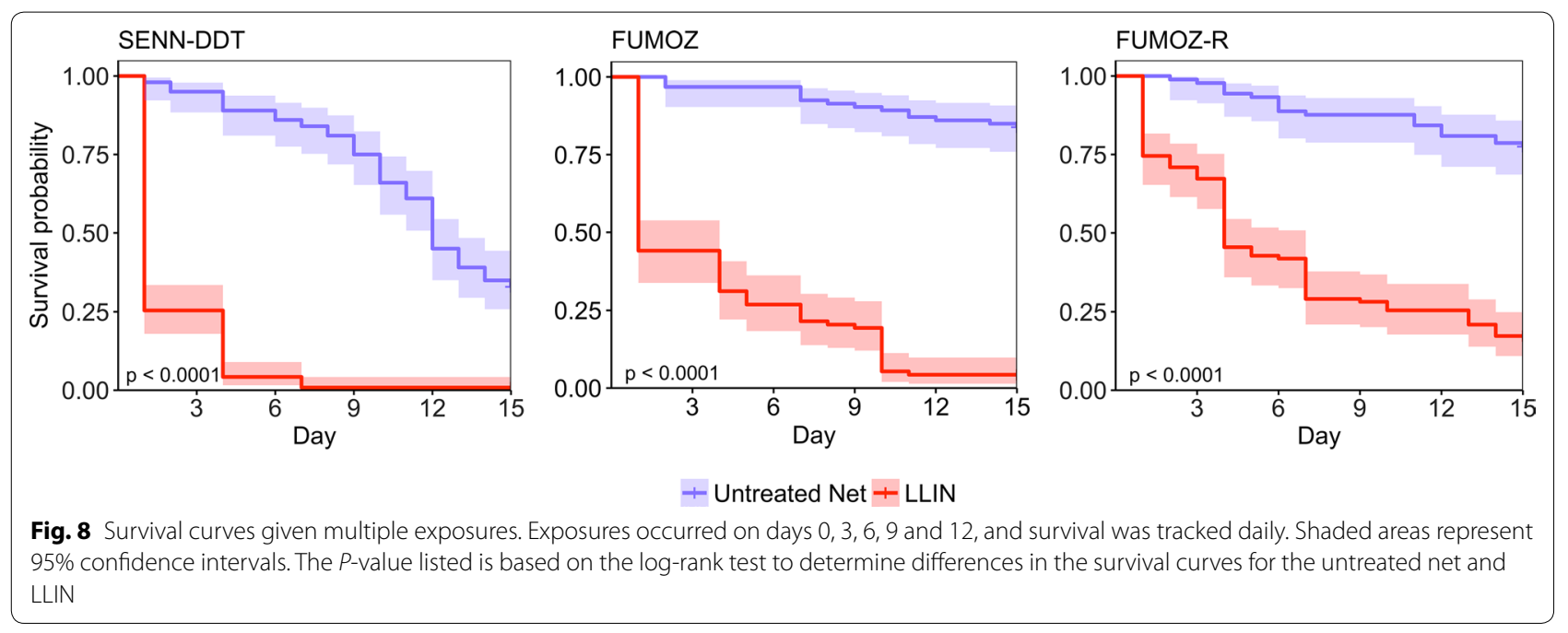

Table 3 Proportion of the population that survived and blood-fed on both initial and final exposure

\begin{tabular}{|c|c|c|c|c|c|c|}
\hline \multirow[t]{2}{*}{ Colony } & \multicolumn{2}{|c|}{ Initial $n$} & \multicolumn{2}{|c|}{$n$ on day 12} & \multicolumn{2}{|c|}{$\begin{array}{l}\text { Individuals that fed on days } 0 \text { and } \\
12 \text { (\% of initial population) }\end{array}$} \\
\hline & LLIN & Untreated net & LLIN & Untreated net & LLIN & Untreated net \\
\hline SENN-DDT & 104 & 105 & 1 & 46 & $1(0.9)$ & $23(21.9)$ \\
\hline FUMOZ & 95 & 101 & 4 & 81 & $3(3.2)$ & $22(21.7)$ \\
\hline FUMOZ-R & 106 & 94 & 20 & 77 & $2(1.8)$ & $20(21.3)$ \\
\hline
\end{tabular}

survived and blood-fed during the initial and final exposures on the untreated net (Fisher's Exact test, $P<0.0001$ for all strains).

\section{Discussion}

Current understanding of the distribution of resistance derives from extensive use of the standardized WHO discriminating dose assay. It is an intuitive assumption that mosquito populations defined as "resistant" to an insecticide will impact the utility of that insecticide in vector control. We used two novel assays to better understand the possible functional significance of resistance: a "tent" assay, which gave mosquitoes ample space to host-seek (and better approximated natural conditions), and a "cup" assay, which placed the mosquitoes in closer proximity to the host. Following a single exposure to a LLIN in the tent assay, two strains that were categorized as $1 \times$ resistant according to the standard WHO tube assays, one $A$. gambiae (TONGS) and the other $A$. arabiensis (SENNDDT), exhibited greater than $90 \%$ mortality 24-hours post-exposure. While there are many differences between our assay and the standard WHO tube test that could produce these results, perhaps the most important factor may be the dose and exposure time. The WHO tube test exposes mosquitoes to insecticide-impregnated papers that are a "diagnostic" dose, i.e. twice the dose required to kill $100 \%$ of a susceptible laboratory population, which is $0.05 \%$ deltamethrin for Anopheles, for an hour. Our assay, on the other hand, used a Permanet 2.0 LLIN, with a much higher dose of $1.8 \mathrm{~g} / \mathrm{kg}(0.18 \%)$, and for up to 20 minutes, with the contact time decided by the individual mosquito. The exact bioavailability of the insecticide on the LLIN surface is unclear, but given the potential increase in effective dose, it is perhaps not a surprise that the mosquitoes characterized as $1 \times$ resistant died in our assay. While we cannot directly compare our assay with the WHO tube test, we want to highlight that the WHO test tells us very little about what a mosquito may be experiencing in the field, so resistance results abstracted from the assay might not actually correlate to its mortality when it encounters a bednet. This is not a criticism of the WHO tests as they are not designed to estimate the functional impact of resistance. Nonetheless, from an operational perspective, there is a need to understand the epidemiological significance of resistance.

Interestingly, the $1 \times$ population SENN-DDT had only $80 \%$ mortality in the cup assay, consistent with the WHO assay results. These findings are likely due to blood-feeding; individuals that blood-fed during exposure in both assays had significantly lower mortality 
than those that did not, and more individuals blood-fed in the cup assay than in the tent assay. In fact, $100 \%$ of non-blood-fed individuals died following exposure in both assays. The $5 \times$ and $10 \times$ resistant mosquito strains used in both assays showed this effect too (susceptible strains died regardless), suggesting that blood-feeding potentially rescues resistant individuals from mortality following insecticide exposure. Other studies have also shown that blood-fed females had greater survival than non-fed females in the standard WHO assays [29, 38, 39], with multiple blood-feeding enhancing the effect [28]. If blood-feeding does indeed have a "rescue effect" on mortality when exposed to insecticides, as our data suggest, then this could be problematic for malaria transmission given that only blood-fed females can acquire the parasite.

However, we also found evidence of blood-feeding inhibition due to the LLIN, which could temper the effect of decreased mortality with a blood meal. In four of the seven strains tested (SENN, SENN-DDT, TONGS and FANG), a lower proportion of mosquitoes that contacted the net blood-fed when exposed to an LLIN than to an untreated net. Studies have shown that deltamethrin-treated nets reduced blood-feeding of Anopheles, suggesting that this inhibition could be due to contact irritancy $[37,40]$. In a similar study to ours, Glunt et al. [11] also found blood-feeding inhibition in SENN-DDT $(1 \times)$, FUMOZ $(5 \times)$, and FUMOZ-R $(10 \times)$ when mosquitoes were given access to a host after LLIN exposure (and not during exposure). Surprisingly, our results show a much higher feeding compliance in FUMOZ-R on the LLIN than on the untreated net in the tent assay, for which we do not have a good explanation. Studies have found that Anopheles with the knock down resistance $(k d r)$ allele are more attracted to a LLIN than an untreated net [41, 42], but FUMOZ$\mathrm{R}$ and FUMOZ do not have $k d r$ as their resistance is driven by metabolic mechanisms only (see Venter et al. [34] for details). Perhaps these mechanisms also cause an attraction to pyrethroids, though we are not aware of research on this subject.

In both assays, the $5 \times($ FUMOZ) and $10 \times($ FUMOZR) resistant strains survived a single exposure extremely well. Their survival is not just because only $50-60 \%$ contacted the LLIN; the individuals that blood-fed, which necessarily contacted the net, survived better than their non-fed counterparts. Additionally, the susceptible strains, KGB and FANG, had similar relatively low contact rates with the LLIN, yet $97-100 \%$ of them died. The mortality of those mosquitoes that did not contact the net for the susceptible strains could be due to either a spatial effect of the LLIN (for which we can find little information in the literature), or because when we were removing mosquitoes from the assay, it prompted mosquito flight and those that did not previously encounter the net ended up contacting it during this time.

Even though the $5 \times$ and $10 \times$ populations survived a single LLIN exposure well, and despite the potential "rescue effect" of blood-feeding, our results suggest that it is possible that multiple exposures drive down survival sufficiently to substantially impede transmission potential. Malaria transmission depends on the survival of the mosquito from the time it becomes infected during a blood meal to beyond the extrinsic incubation period (EIP) of the parasite. Given a single exposure, none of the $1 \times$-resistant females survived the typical 10-14 day EIP, meaning that they would be unable to transmit parasites. The $5 \times$-resistant females exposed to the LLIN survived the EIP, but significantly less so than on the untreated net, suggesting that the insecticide-treated net is still having some effect beyond a simple barrier. In contrast, the vast majority of the $10 \times$-resistant females survived the EIP with no difference between the LLIN and untreated net, indicating that the single insecticide exposure had no effect. However, with multiple exposures simulating repeat contact with an LLIN over sequential feeding cycles (mosquitoes are anticipated to blood-feed every $2-4$ days as this is the duration of the gonotrophic cycle), all strains suffered significant mortality regardless of resistance, and those mosquitoes that survived the EIP exhibited a reduction in blood-feeding, translating to greatly reduced malaria transmission potential.

In summary, our results suggest that realistic contact with an LLIN imposes substantial mortality against $1 \times$-resistant mosquitoes and hence, this level of resistance might have negligible impact on LLIN efficacy in the field. Moreover, with multiple exposures, even $10 \times$-resistant populations suffer greater mortality and reduced blood-feeding on an LLIN compared to an untreated net, suggesting that LLINs are still likely to contribute to control in areas of more intense resistance. We acknowledge that we have used only a limited number of mosquito strains and one type of LLIN, and populations in the field could exhibit a greater range of resistance mechanisms and intensities to different insecticides [43]. Additionally, we used an unused, unwashed LLIN to be consistent with other resistance assays, which also guaranteed maximum efficacy. Data on durability of nets is somewhat mixed: some studies show PermaNet 2.0 to lose efficacy after 5 washes [44], after 15 washes [45], or potentially to show no significant change in efficacy with washing and/or use $[46,47]$. However, it might be expected that decay in the active ingredient and/or loss of net integrity will exacerbate the functional effects of resistance. As such, extending the type of research presented here to nets of different age and use history would 
be an important next step to examine potential interactions between resistance intensity and net durability. Our simplified assay systems also potentially masked more complex mosquito behaviors that could play a role in nature [35]. Nonetheless, in line with the recommendation of WHO [21] and others [2, 3], our study highlights the need for more data on the intensity of resistance, not only to fully understand the functional significance of resistance but also to manage or mitigate the problem.

Next generation LLINs are close to entering operational use. These nets are designed to overcome pyrethroid resistance, either through use of the synergist piperonyl butoxide (PBO), which reduces the capacity of mosquitoes to detoxify the insecticide, or through the addition of alternative insecticides with modes of action distinct from pyrethroids. As a side experiment, we tested one of these next generation LLINs (Permanet 3.0, which combines deltamethrin with PBO) against our resistant strains using the cup assay (see Additional file 1: Text S1 for brief description and summary of results). All strains, including the $10 \times$ strain, suffered $100 \%$ mortality within 24 hours on the portion of the net containing $\mathrm{PBO}$ (Additional file 2: Figure S1). Mosquitoes also bloodfed less on the PBO portion of the Permanet 3.0 compared to the side of the Permanet 3.0 without PBO (Additional file 3: Figure S2) and suffered 100\% mortality regardless of bloodfed status (Additional file 4: Figure S3). Other studies have shown broad-scale efficacy of PBO nets [48], with only limited evidence of PBO net failure [49]. At present, these nets are more costly than traditional pyrethroid-only nets, so it would be beneficial to be able to target their distribution into areas where insecticide resistance is most critical. Targeting areas on the basis of $1 \times$ resistance has little strategic value as $1 \times$ tells us little about the functional significance of resistance. Based on our results, the distribution of $10 \times$ resistance could be more informative to prioritize areas, but unfortunately the data on resistance intensity are very limited [22]. For example, the World Health Organization's Malaria Threats Map of Vector Insecticide Resistance [22] includes 788 entries for tests conducted using the diagnostic dose of pyrethroid insecticide on Anopheles mosquitoes for Kenya alone, yet only 10 tests conducted using the intensity bioassay. In other countries, like Sudan, there are simply no data on resistance intensity despite an abundance of data on the diagnostic concentration (756 studies) [22]. Collecting resistance data is a challenge for many national malaria control programmes, but where this is possible, data on resistance intensity could prove valuable to aid in the strategic distribution of next-generation LLINs, which will almost certainly be a key tool for malaria control in the face of increasing resistance.

\section{Conclusions}

Why has resistance not caused a clear failure of malaria control? The results of this study suggest that the current assays may not be characterizing resistance adequately. We found that mortality following LLIN exposure depends on the type of exposure, the blood-feeding status of the female, and the frequency of exposure, all of which are not accounted for in the current WHO tube assay. Furthermore, we found a distinct difference in survival between $1 \times-, 5 \times$ - and $10 \times$-resistant populations, suggesting that intensity of resistance matters. Regardless of resistance intensity, however, we found that the LLIN was still more effective than an untreated net and given multiple exposures, even a $10 \times$-resistant population suffered significant mortality. While the current WHO assays are a convenient starting point for assessing resistance in the field, new assays need to be developed to better characterize resistance in terms of transmission potential. Gaining a better understanding of what resistance means functionally for transmission will ultimately lead to more efficient vector control strategies.

\section{Supplementary information}

Supplementary information accompanies this paper at https://doi. org/10.1186/s13071-020-04055-9.

Additional file 1: Text S1. Test of the Permanet 3.0 using the Cup assay.

Additional file 2: Figure S1. Mortality 24-h post-exposure. The bars show the mean $( \pm S E$ ) proportion of mosquitoes that died following exposure to either the side of the Permanet 3.0 (red bars) or top of the Permanet 3.0 (blue bars).

Additional file 3: Figure S2. Blood-feeding behavior of mosquitoes. The bars show the mean ( \pm SE) proportion of mosquitoes that successfully took a blood meal from a human host arm through either the side of the Permanet 3.0 (red bars) or the top of the Permanet 3.0 (blue bars).

Additional file 4: Figure S3. Mortality 24-h post-exposure based on blood-fed status. The bars show the mean ( \pm SE) proportion of mosquitoes that died following either a successful or unsuccessful attempt to blood-feed on a human host arm while being exposed either the top of the Permanet 3.0 (left panel) or the side of the Permanet 3.0 (right panel).

\section{Abbreviations}

LLIN: long-lasting insecticidal net; WHO: World Health Organization; CDC: Centers for Disease Control and Prevention.

\section{Acknowledgements}

We would like to thank the staff at the Richard Hunt Insectary at the National Institute for Communicable Diseases in Johannesburg, South Africa, for their assistance in colony maintenance.

\section{Authors' contributions}

MKG and MBT designed the study. MKG and SVO conducted the experiments. MKG analyzed the data. MKG and MBT wrote the manuscript. All authors read and approved the final manuscript.

\section{Funding}

This study was part supported by NIH NIAID Grant no. R21 Al113609-01A1 and the USDA NIFA and Hatch Appropriations under Project no. PEN04691 and Accession no. 1018545. The funders had no role in study design, data collection and analysis, decision to publish or preparation of the manuscript. 


\section{Availability of data and materials}

The datasets supporting the conclusions of this article are included within the article and its additional file.

\section{Ethics approval and consent to participate}

All experimental methods were consistent with Penn State IBC protocol no. 48219. The Pennsylvania State University Institutional Review Board determined that the experiments whereby uninfected mosquitoes were attracted to a host's arm did not meet the criteria for human subjects research.

\section{Consent for publication}

Not applicable.

\section{Competing interests}

The authors declare that they have no competing interests.

\section{Author details}

${ }^{1}$ Department of Entomology, Pennsylvania State University, University Park, PA, USA. ${ }^{2}$ Centre for Emerging Zoonotic and Parasitic Diseases, National Institute for Communicable Diseases of the National Health Laboratory Service, Johannesburg, South Africa. ${ }^{3}$ Wits Research Institute for Malaria, MRC Collaborating Centre for Multi-disciplinary Research on Malaria, School of Pathology, Faculty of Health Sciences, University of the Witwatersrand, Johannesburg, South Africa.

Received: 4 December 2019 Accepted: 30 March 2020

Published online: 07 April 2020

\section{References}

1. Bhatt S, Weiss DJ, Cameron E, Bisanzio D, Mappin B, Dalrymple U, et al. The effect of malaria control on Plasmodium falciparum in Africa between 2000 and 2015. Nature. 2015:526:207-11.

2. Hemingway J, Ranson H, Magill A, Kolaczinski J, Fornadel C, Gimnig J, et al. Averting a malaria disaster: will insecticide resistance derail malaria control? Lancet. 2016;387:1785-8.

3. Ranson H, Lissenden N. Insecticide resistance in African Anopheles mosquitoes: a worsening situation that needs urgent action to maintain malaria control. Trends Parasitol. 2016;32:187-96.

4. Protopopoff N, Mosha JF, Lukole E, Charlwood JD, Wright A, Mwalimu $C D$, et al. Effectiveness of a long-lasting piperonyl butoxide-treated insecticidal net and indoor residual spray interventions, separately and together, against malaria transmitted by pyrethroid-resistant mosquitoes: a cluster, randomised controlled, two-by-two factorial design trial. Lancet. 2018;391:1577-88.

5. Bradley J, Ogouyèmi-Hounto A, Cornélie S, Fassinou J, de Tove YSS, Adéothy AA, et al. Insecticide-treated nets provide protection against malaria to children in an area of insecticide resistance in southern Benin. Malar J. 2017;16:225.

6. Strode C, Donegan S, Garner P, Enayati AA, Hemingway J. The impact of pyrethroid resistance on the efficacy of insecticide-treated bed nets against African anopheline mosquitoes: systematic review and metaanalysis. PLoS Med. 2014;11:e1001619.

7. Ochomo E, Chahilu M, Cook J, Kinyari T, Bayoh NM, West P, et al. Insecticide-treated nets and protection against insecticide-resistant malaria vectors in western Kenya. Emerg Infect Dis. 2017;23:758-64.

8. Chourasia MK, Kamaraju R, Kleinschmidt I, Bhatt RM, Swain DK, Knox TB, et al. Impact of long-lasting insecticidal nets on prevalence of subclinical malaria among children in the presence of pyrethroid resistance in Anopheles culicifacies in Central India. Int J Infect Dis. 2017;57:123-9.

9. Kleinschmidt I, Bradley J, Knox TB, Mnzava AP, Kafy HT, Mbogo C, et al. Implications of insecticide resistance for malaria vector control with longlasting insecticidal nets: a WHO-coordinated, prospective, international, observational cohort study. Lancet Infect Dis. 2018;18:640-9.

10. Churcher TS, Lissenden N, Griffin JT, Worrall E, Ranson H. The impact of pyrethroid resistance on the efficacy and effectiveness of bednets for malaria control in Africa. Elife. 2016;5:e16090

11. Glunt KD, Coetzee M, Huijben S, Koffi AA, Lynch PA, N'Guessan R, et al. Empirical and theoretical investigation into the potential impacts of insecticide resistance on the effectiveness of insecticide-treated bed nets. Evol Appl. 2017;11:431-41.

12. Thomas MB, Read AF. The threat (or not) of insecticide resistance for malaria control. Proc Natl Acad Sci USA. 2016;113:8900-2.

13. Kristan M, Lines J, Nuwa A, Ntege C, Meek SR, Abeku TA. Exposure to deltamethrin affects development of Plasmodium falciparum inside wild pyrethroid resistant Anopheles gambiae s.s. mosquitoes in Uganda. Parasites Vectors. 2016;9:100.

14. Alout H, Yameogo B, Djogbenou LS, Chandre F, Dabire RK, Corbel V, et al. Interplay between Plasmodium infection and resistance to insecticides in vector mosquitoes. J Infect Dis. 2014;210:1464-70.

15. Alout H, Ndam NT, Sandeu MM, Djegbe I, Chandre F, Dabiré RK, et al. Insecticide resistance alleles affect vector competence of Anopheles gambiae s.s. for Plasmodium falciparum field isolates. PLoS ONE. 2013;8:e63849.

16. Alout H, Dabire RK, Djogbenou LS, Abate L, Corbel V, Chandre F, et al. Interactive cost of Plasmodium infection and insecticide resistance in the malaria vector Anopheles gambiae. Sci Rep. 2016;6:29755.

17. Assogba BS, Milesi P, Djogbenou LS, Berthomieu A, Makoundou P, BabaMoussa LS, et al. The ace-1 locus Is amplified in all resistant Anopheles gambiae mosquitoes: fitness consequences of homogeneous and heterogeneous duplications. PLoS Biol. 2016;14:e2000618.

18. Kliot A, Ghanim M. Fitness costs associated with insecticide resistance Pest Manag Sci. 2012;68:1431-7.

19. Okoye PN, Brooke BD, Hunt RH, Coetzee M. Relative developmental and reproductive fitness associated with pyrethroid resistance in the major southern African malaria vector, Anopheles funestus. Bull Entomol Res. 2007;97:599-605

20. Collins E, Vaselli NM, Sylla M, Beavogui AH, Orsborne J, Lawrence G, et al. The relationship between insecticide resistance, mosquito age and malaria prevalence in Anopheles gambiae s.l. from Guinea. Sci Rep. 2019;9:8846.

21. WHO. Test procedures for insecticide resistance monitoring in malaria vector mosquitoes. 2nd ed. Geneva: World Health Organization; 2016

22. IR mapper. http://www.irmapper.com. Accessed 5 Sept 2019.

23. WHO Malaria Threats Map: tracking biological challenges to malaria control and elimination. http://www.who.int/malaria/maps/threats/). Accessed 5 Sept 2019.

24. Coleman M, Hemingway J, Gleave KA, Wiebe A, Gething PW, Moyes CL. Developing global maps of insecticide resistance risk to improve vector control. Malar J. 2017;16:86

25. Jones CM, Sanou A, Guelbeogo WM, Sagnon N, Johnson PCD, Ranson $\mathrm{H}$. Aging partially restores the efficacy of malaria vector control in insecticide-resistant populations of Anopheles gambiae s.l. from Burkina Faso. Malar J. 2012;11:24.

26. Chouaibou MS, Chabi J, Bingham GV, KnoxTB, N'dri L, Kesse NB, et al. Increase in susceptibility to insecticides with aging of wild Anopheles gambiae mosquitoes from Côte d'Ivoire. BMC Inf Dis. 2012;12:214.

27. Rajatileka S, Burhani J, Ranson H. Mosquito age and susceptibility to insecticides. Trans R Soc Trop Med Hyg. 2011;105:247-53.

28. Oliver SV, Brooke BD. The effect of multiple blood-feeding on the longevity and insecticide resistant phenotype in the major malaria vector Anopheles arabiensis (Diptera: Culicidae). Parasites Vectors. 2014;7:390.

29. Machani MG, Ochomo E, Sang D, Bonizzoni M, Zhou G, Githeko AK, et al. Influence of blood meal and age of mosquitoes on susceptibility to pyrethroids in Anopheles gambiae from Western Kenya. Malar J. 2019;18:112.

30. Viana M, Hughes A, Matthiopoulos J, Ranson H, Ferguson HM. Delayed mortality effects cut the malaria transmission potential of insecticideresistant mosquitoes. Proc Natl Acad Sci USA. 2016;113:8975-80.

31. WHO. Guidelines for laboratory and field-testing of long-lasting insecticidal nets. Geneva: World Health Organization; 2013.

32. Massue DJ, Lorenz LM, Moore JD, Ntabaliba WS, Ackerman S, Mboma ZM, et al. Comparing the new Ifakara Ambient Chamber Test with WHO cone and tunnel tests for bioefficacy and non-inferiority testing of insecticidetreated nets. Malar J. 2019;18:153.

33. Hunt RH, Brooke BD, Pillay C, Koekemoer LL, Coetzee M. Laboratory selection for and characteristics of pyrethroid resistance in the malaria vector Anopheles funestus. Med Vet Entomol. 2005;19:271-5.

34. Venter N, Oliver SV, Muleba M, Davies C, Hunt RH, Koekemoer LL, et al. Benchmarking insecticide resistance intensity bioassays for Anopheles 
malaria vector species against resistance phenotypes of known epidemiological significance. Parasites Vectors. 2017;10:198.

35. Parker JEA, Angarita-Jaimes N, Abe M, Towers CE, Towers D, McCall PJ. Infrared video tracking of Anopheles gambiae at insecticide-treated bed nets reveals rapid decisive impact after brief localised net contact. Sci Rep. 2015;5:13392.

36. Ohm JR, Baldini F, Barreaux P, Lefevre T, Lynch PA, Suh E, et al. Rethinking the extrinsic incubation period of malaria parasites. Parasites Vectors. 2018:11:178.

37. Malima RC, Oxborough RM, Tungu PK, Maxwell C, Lyimo I, Mwingira V, et al. Behavioural and insecticidal effects of organophosphate-, carbamate- and pyrethroid-treated mosquito nets against African malaria vectors. Med Vet Entomol. 2009;23:317-25.

38. Spillings BL, Coetzee M, Koekemoer LL, Brooke BD. The effect of a single blood meal on the phenotypic expression of insecticide resistance in the major malaria vector Anopheles funestus. Malar J. 2008;7:226.

39. Halliday WR, Feyereisen R. Why does DDT toxicity change after a blood meal in adult female Culex pipiens? Pest Biochem Physiol. 1987;28:172-81.

40. Chandre F, Darriet F, Duchon S, Finot L, Manguin S, Carnevale P, et al. Modifications of pyrethroid effects associated with $k d r$ mutation in Anopheles gambiae. Med Vet Entomol. 2000;14:81-8.

41. Porciani A, Diop M, Moiroux N, Kadoke-Lambi T, Cohuet A, Chandre F, et al. Influence of pyrethroïd-treated bed net on host seeking behavior of Anopheles gambiae s.s. carrying the $k d r$ allele. PLOS ONE. 2017;12:e0164518-15.

42. Kawada H, Ohashi K, Dida GO, Sonye G, Njenga SM, Mwandawiro C, et al. Insecticidal and repellent activities of pyrethroids to the three major pyrethroid-resistant malaria vectors in western Kenya. Parasites Vectors. 2014;7:208.

43. Hancock PA, Wiebe A, Gleave KA, Bhatt S, Cameron E, Trett A, et al. Associated patterns of insecticide resistance in field populations of malaria vectors across Africa. Proc Natl Acad Sci USA. 2018;115:5938-43.
44. Atieli FK, Munga SO, Ofulla AV, Vulule JM. The effect of repeated washing of long-lasting insecticide-treated nets (LLINs) on the feeding success and survival rates of Anopheles gambiae. Malar J. 2010;9:304.

45. Agossa FR, Padonou GG, Gnanguenon V, Oké-Agbo F, Zola-Sahossi J, Dègnonvi $\mathrm{H}$, et al. Laboratory and field evaluation of the impact of washings on the effectiveness of LifeNet ${ }^{\circledR}$, Olyset ${ }^{\circledR}$ and PermaNet ${ }^{\circledR} 2.0$ in two areas, where there is a high level of resistance of Anopheles gambiae to pyrethroids, Benin, West Africa. Malar J. 2014;13:193.

46. Msangi S, Lyatuu E, Masenga C, Kihumo E. The effects of washing and duration of use of long-lasting insecticidal nets (PermaNets) on insecticidal effectiveness. Acta Trop. 2008;107:43-7.

47. Graham K, Kayedi MH, Maxwell C, Kaur H, Rehman H, Malima R, et al. Multi-country field trials comparing wash-resistance of PermaNet and conventional insecticide-treated nets against anopheline and culicine mosquitoes. Med Vet Entomol. 2005;19:72-83.

48. Gleave K, Lissenden N, Richardson M, Choi L, Ranson H. Piperonyl butoxide (PBO) combined with pyrethroids in insecticide-treated nets to prevent malaria in Africa. Cochrane Database Syst Rev. 2018;11:CD012776.

49. Riveron JM, Huijben S, Tchapga W, Tchouakui M, Wondji MJ, Tchoupo M, et al. Escalation of pyrethroid resistance in the malaria vector Anopheles funestus induces a loss of efficacy of piperonyl butoxide-based insecticide-treated nets in Mozambique. J Infect Dis. 2019;220:467-75.

\section{Publisher's Note}

Springer Nature remains neutral with regard to jurisdictional claims in published maps and institutional affiliations.
Ready to submit your research? Choose BMC and benefit from:

- fast, convenient online submission

- thorough peer review by experienced researchers in your field

- rapid publication on acceptance

- support for research data, including large and complex data types

- gold Open Access which fosters wider collaboration and increased citations

- maximum visibility for your research: over $100 \mathrm{M}$ website views per year

At BMC, research is always in progress.

Learn more biomedcentral.com/submissions 